\title{
Low Damage Etching of GaN Surfaces via Bias-Assisted Photoenhanced Electrochemical Oxidation in Deionized Water
}

\author{
EMRE ALPTEKIN ${ }^{1}$ HONGBO YU, ${ }^{3}$ EKMEL OZBAY $, 1,2,3$ \\ and OZGUR AKTAS ${ }^{1,3,4}$
}

1.-Department of Electrical and Electronics Engineering, Bilkent University, Bilkent, Ankara, Turkey. 2.-Department of Physics, Bilkent University, Bilkent, Ankara, Turkey. 3.-Nanotechnology Research Center, Bilkent University, Bilkent, Ankara, Turkey. 4.-e-mail: aktas @ee.bilkent.edu.tr

Properties of GaN surfaces etched by bias-assisted photoenhanced electrochemical (PEC) oxidation in deionized water and subsequent removal of the oxidized material are investigated using Schottky diodes fabricated on etched surfaces. It is demonstrated that with a short anneal at $700^{\circ} \mathrm{C}$ after removal of the oxide, it is possible to obtain a low damage surface with near ideal breakdown and capacitance-voltage (C-V) characteristics. Good quality Schottky diodes are fabricated on surfaces etched as much as $120 \mathrm{~nm}$. The undercutting of masked surfaces is also demonstrated. Thus, given the band-gap selectivity, the possibility to undercut masked areas, and the low damage surfaces that can be obtained, the process demonstrated in this paper is suitable for use in fabrication of self-aligned GaN bipolar transistor structures.

Key words: GaN etching, electrochemical oxidation, self-aligned process

\section{INTRODUCTION}

Techniques for low-damage etching of $\mathrm{GaN}$ and its alloys are important for optimization of devices such as high electron mobility transistors and heterojunction bipolar transistors (HBTs) ${ }^{1-4}$ Ultraviolet photoenhanced electrochemical (PEC) etching and oxidation of $\mathrm{GaN}$ is a versatile method that is both band-gap and doping selective. ${ }^{5-8}$ The PEC etching can achieve a smooth surface with low etch damage. $^{9,10}$ A process similar to PEC etching and oxidation of GaN in alkaline solutions is the biasassisted photoenhanced electrochemical oxidation of GaN in deionized water (BPECO/DI). ${ }^{11}$ In this approach, the oxide that forms on the surface remains intact and thick oxide layers can be obtained. To achieve etching of the GaN surface, the oxide grown by BPECO/DI can be removed by an hydrochloric acid $(\mathrm{HCl})$ or hydrofloric acid $(\mathrm{HF})$ etch following the oxidation. In this paper, the etching process employing bias-assisted photoenhanced electrochemical oxidation in deionized water and a

(Received March 7, 2006; accepted September 25, 2006; published online January 9, 2007) subsequent acid bath removal of the oxide is called "BPECO/DI etch."

Similar to the PEC etching in alkaline solutions, the BPECO/DI etching process is attractive due to the band gap and $n / p$ doping selectivity that can be achieved. However, the process presented here has the additional advantage that the BPECO/DI oxidation and etching can be performed with a photoresist mask in place and also without damaging existing ohmic or Schottky metallization on the sample. Catalytic titanium masks are not needed for BPECO/DI etching, and the process can be applied to relatively large areas. Hence, the process does not suffer from the inhomogeneities commonly observed close to the titanium mask edges used with PEC and bias-assisted PEC (BPEC) processes. Thus, BPECO/ DI etching has the potential to be a technique that is more widely applied than PEC etching.

The lack of a low damage etching process that is compatible with the rest of the fabrication steps has been a factor hindering GaN HBT fabrication. For the fabrication of GaN HBTs, an n/p doping selective etch process that does not result in damaged surfaces after $100 \mathrm{~nm}$ to $150 \mathrm{~nm}$ etching is necessary. 
In this paper, we demonstrate that BPECO/DI etching yields low-damage surfaces after etching as much as $120 \mathrm{~nm}$. A postetch anneal at $700^{\circ} \mathrm{C}$ is required to improve the surface characteristics to near ideal. The properties of surfaces oxidized and etched by BPECO/DI are investigated using Schottky diodes fabricated on etched surfaces and also by using Auger electron spectroscopy (AES) and atomic force microscope (AFM) measurements. The results presented here should motivate further investigation of BPECO/DI for use in GaN HBT processing.

\section{EXPERIMENTAL}

The layer structure used in this study consisted of a $0.5-\mu \mathrm{m}$ undoped $\mathrm{u}-\mathrm{GaN}$ layer grown on top of a $0.5-\mu \mathrm{m} \mathrm{n}+\mathrm{GaN}$ (intended silicon doping $3 \mathrm{e} 18 \mathrm{~cm}^{-3}$ ) layer at the bottom. All samples were grown on sapphire substrates using metal organic chemical vapor deposition. Before the oxidation of the samples with BPECO/DI, mesa etching and ohmic contact fabrication steps were completed. The ohmic contact metallization was $\mathrm{Ti} / \mathrm{Al} / \mathrm{Ni} / \mathrm{Au}(25 / 200 / 40 /$ $50 \mathrm{~nm}$ ) thermally evaporated, patterned by lift-off, and annealed at $750^{\circ} \mathrm{C}$ for $30 \mathrm{~s}$. The mesa structures were needed for establishing ohmic contacts to the $\mathrm{n}+\mathrm{GaN}$ layer. A few of the mesa structures were partially masked with sputtered silicon nitride (SiN) to enable etch depth measurement. For the collection of photogenerated electrons, 0.5 -mm-wide ohmic contact metallization was fabricated on the four edges of the samples.

The BPECO/DI oxidation was performed using the method in Ref. 11. The light from a $200 \mathrm{~W} \mathrm{Hg-Xe}$ ultraviolet lamp was focused by an elliptical mirror, and the sample was placed close to the focus point. The oxidation time was limited by the thickness of the undoped layer, and the maximum oxidation time was selected to be $3 \mathrm{~h}$ after preliminary tests.

In order to measure the BPECO/DI oxide thickness, the top of the mesa structures was patterned by photoresist following BPECO/DI oxidation, and the oxide was etched from the exposed areas by a 1:5 $\mathrm{HCl}$ :DI etch. After measuring the oxide thickness from the exposed edge by using a profilometer, all of the remaining oxide on the surface was etched. Next, in order to measure the etch depth, the SiN mask was removed by a 1:100 HF:DI etch and the etch depth was measured from the exposed edge on top of the mesa structures. Some of the samples were annealed at $700^{\circ} \mathrm{C}$ for $30 \mathrm{~s}$ under nitrogen ambient in an effort to remove the BPECO/DI etch damage. As a final step, Ni/Au $(45 / 50 \mathrm{~nm})$ Schottky structures were patterned and evaporated on the mesas. Immediately before loading samples into the evaporator for $\mathrm{Ni} / \mathrm{Au}$ evaporation, the samples were dipped in 1:2 HCl:DI solution for $30 \mathrm{~s}$, rinsed, and blown dry with nitrogen. The AES and AFM measurements were taken from bare samples without mesas and Schottky structures, but with the BPECO/DI contact metallization in place on four edges of the sample. Capacitance-voltage (C-V) measurements were done using an HP-4284 LCR meter at $100 \mathrm{kHz}$. The AES measurements were performed on a PHI 660 scanning Auger microprobe. The AES spectra were taken from a $5 \times 5 \mu \mathrm{m}$ area on the surface. The AFM measurements were taken on a Digital Instruments Multimode system.

\section{RESULTS}

Etch depth versus time and voltage data obtained as described above are given in Table I. The etch depths and oxide thicknesses given in Table I were measured from the top of the mesa structures, as noted above. In earlier work, ${ }^{11}$ saturation of oxide thickness with time was observed to set in after $1 \mathrm{~h}$ of oxidation, and the saturated oxide thickness reported at $5 \mathrm{~V}$ bias was $175 \mathrm{~nm}$. In the present study, a similar saturation of oxide thickness is also observed. However, the saturation of oxide thickness occurs after $2 \mathrm{~h}$ of BPECO/DI oxidation, and the resulting oxide thickness is much larger. Crucially, it needs to be noted that the etch-depth saturation is not observed at the maximum feasible etching time, despite the strong saturation of the oxide thickness. Otherwise, the etch rate increases with increasing anode bias, and in general stays in the same range as the values reported in earlier work. ${ }^{11}$

The current-voltage characteristics of Schottky contacts fabricated on surfaces with various treatments are summarized in Table II. Figure 1 shows the reverse current characteristics of these devices. From these results, we see that the BPECO/DI etching causes a reduction in breakdown voltage (defined as the voltage at which the reverse current increase to $1 \mathrm{~mA}$ ) and an increase in the ideality factor. The breakdown voltages are higher for samples that were annealed after the removal of the BPECO/DI oxide. Under certain conditions, breakdown voltages after annealing increase back to a value that is expected from the decrease in the undoped layer thickness. From Table II, it is also observed that annealing does not result in a significant change in the ideality factor of the BPECO/DI etched Schottky diodes.

The increase in the reverse leakage current with BPECO/DI etching and the subsequent reduction of the leakage current with annealing in $\mathrm{N}_{2}$ can be

\section{Table I. Oxide Thicknesses and Etch Depths} Measured at Different Oxidation Conditions.

\begin{tabular}{|c|c|c|c|}
\hline $\begin{array}{l}\text { Voltage } \\
\text { (V) }\end{array}$ & $\begin{array}{l}\text { Time } \\
\text { (min) }\end{array}$ & $\begin{array}{c}\text { Etch Depth } \\
(\text { nm) }\end{array}$ & $\begin{array}{c}\text { Oxide Thickness } \\
\text { (nm) }\end{array}$ \\
\hline 4 & 60 & 50 & 400 \\
\hline 6 & 60 & 65 & 450 \\
\hline 6 & 120 & 130 & 700 \\
\hline 6 & 180 & 220 & 780 \\
\hline 8 & 60 & 120 & 600 \\
\hline
\end{tabular}


Table II. Characteristics of Schottky Contacts Fabricated on GaN Surfaces with Various Treatments; the Estimated Breakdown Voltages are Calculated Assuming a Uniform Field in the Undoped GaN Layer.

\begin{tabular}{|c|c|c|c|c|c|c|}
\hline $\begin{array}{l}\text { Voltage } \\
\text { (V) }\end{array}$ & $\begin{array}{l}\text { Time } \\
\text { (min) }\end{array}$ & $\begin{array}{c}\text { Etch Depth } \\
(\mathbf{n m})\end{array}$ & $\begin{array}{c}\text { Postetch } \\
\text { Treatment }\end{array}$ & $\begin{array}{l}\text { Breakdown } \\
\text { Voltage }\left(V_{B}\right)\end{array}$ & $\begin{array}{l}\text { Ideality } \\
\text { Factor }\end{array}$ & $\underset{(V)}{\text { Estimated }} \mathrm{V}_{\mathbf{B}}$ \\
\hline - & - & 0 & - & -70 & 1.25 & - \\
\hline 4 & 60 & 50 & - & -54 & 1.52 & 63 \\
\hline 4 & 60 & 50 & Annealed & -60 & 1.89 & 63 \\
\hline 6 & 60 & 65 & - & -44 & 1.71 & 61 \\
\hline 6 & 60 & 65 & Annealed & -51 & 1.72 & 61 \\
\hline 6 & 120 & 130 & - & -33 & 1.67 & 52 \\
\hline 6 & 120 & 130 & Annealed & -41 & 1.82 & 52 \\
\hline 8 & 60 & 120 & - & -38 & 1.93 & 52 \\
\hline 8 & 60 & 120 & Annealed & -54 & 1.97 & 52 \\
\hline
\end{tabular}

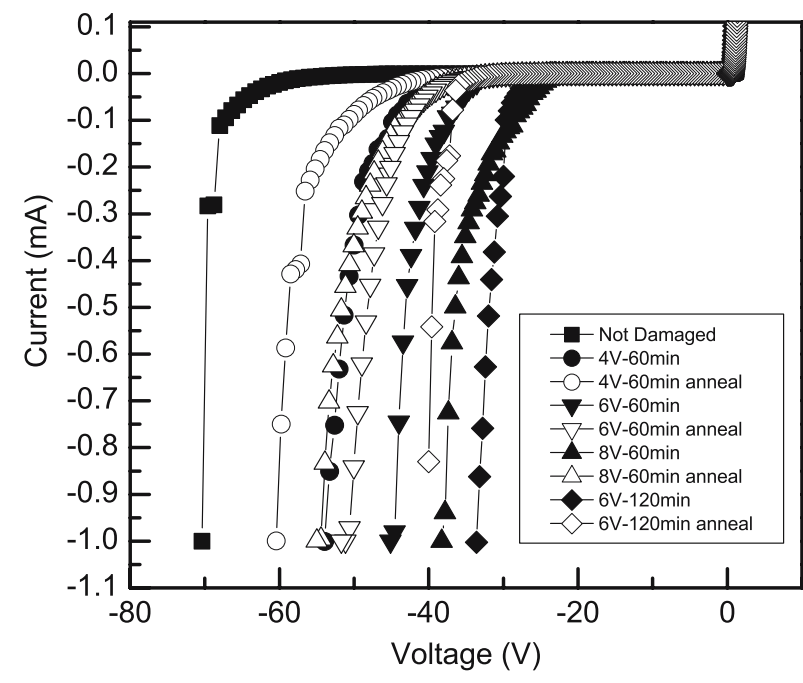

Fig. 1. Reverse I-V characteristics of Schottky contacts fabricated on surfaces after various treatments. The legend shows the PECoxidation voltage and time, and if applied, a postetch $700^{\circ} \mathrm{C}, 30 \mathrm{~s}$ anneal.

attributed to the creation and removal of point defects on or close to the surface. Considering the possible reverse conduction mechanisms, ${ }^{12-14}$ it can be suggested that defects created by BPECO/DI etching result in increased trap-assisted tunneling from the metal into the semiconductor. The reduction in the reverse leakage current can then be related to the reduction in the density of the defects close to the surface. In this picture, the defects created by BPECO/DI etching provide states between the midgap and the conduction band. The increase in the ideality factor with BPECO/DI etching and the insensitivity of the ideality factor to annealing can be either due to an increase in surface nonuniformity (of the Schottky barrier height) or to the presence of an interfacial layer. Also, the nonsaturating reverse characteristics observed in Fig. 1 can be attributed to a voltagedependent effective Schottky barrier height induced by surface nonuniformity or the presence of an interfacial layer.

The C-V characteristics presented in Fig. 2a indicate that the untreated surface has a uniform carrier density profile. After etching of the BPECO/ DI-grown oxide, the surface has a region of low carrier density, as indicated by the high slope observed in Fig. $2 \mathrm{~b}$ for bias voltages close to $0 \mathrm{~V}$. After annealing, the $\mathrm{C}-\mathrm{V}$ characteristics shown in Fig. 2c approach that of an untreated surface shown in Fig. 2a. These $\mathrm{C}-\mathrm{V}$ characteristics indicate that BPECO/DI etching results in creation of compensating defects close to the surface.

Given the information about the point defects in $\mathrm{GaN},{ }^{15,16}$ it may be concluded that BPECO/DI oxidation results in creation of gallium vacancies $\left(\mathrm{V}_{\mathrm{Ga}}\right)$ and incorporation of oxygen to nitrogen sites $\left(\mathrm{O}_{\mathrm{N}}\right)$. It is likely that these two defects are bound together, forming $\mathrm{V}_{\mathrm{Ga}}-\mathrm{O}_{\mathrm{N}}$ complexes. ${ }^{16}$ Previous studies based on photoluminescence investigation of GaN etched by PEC in potassium hydroxide $(\mathrm{KOH})$ solution have reached the same conclusion. ${ }^{17}$

The fact that near-ideal breakdown voltages and $\mathrm{C}-\mathrm{V}$ characteristics were obtained on 120-nm etched surfaces after a $700^{\circ} \mathrm{C} 30 \mathrm{~s}$ anneal indicates that the damage induced by BPECO/DI is shallow and can easily be removed. In contrast, surfaces etched by reactive ion etching (RIE) by only $10 \mathrm{~nm}$ using the best techniques require an $850^{\circ} \mathrm{C}, 30 \mathrm{~s}$ anneal for damage removal. Deeper RIE etches require etching of the sample by $\mathrm{KOH}$ for damage removal, making the process unusable for practical purposes. Similarly, the PEC process is not suitable for use in self-aligned emitter-base etching of GaN HBT structures, because PEC employs $\mathrm{KOH}$ during the etching process, which needs to be performed at low etching rates to achieve low damage. Thus, etching of GaN using the BPECO/DI approach stands out as a unique tool for deep etching without creating extensive surface damage. Such a process is critically needed for GaN-based HBT fabrication.

The AFM scans indicate that the BPECO/DI etched $\mathrm{GaN}$ surface is rougher than the original unetched surface. The root-mean-square (RMS) 

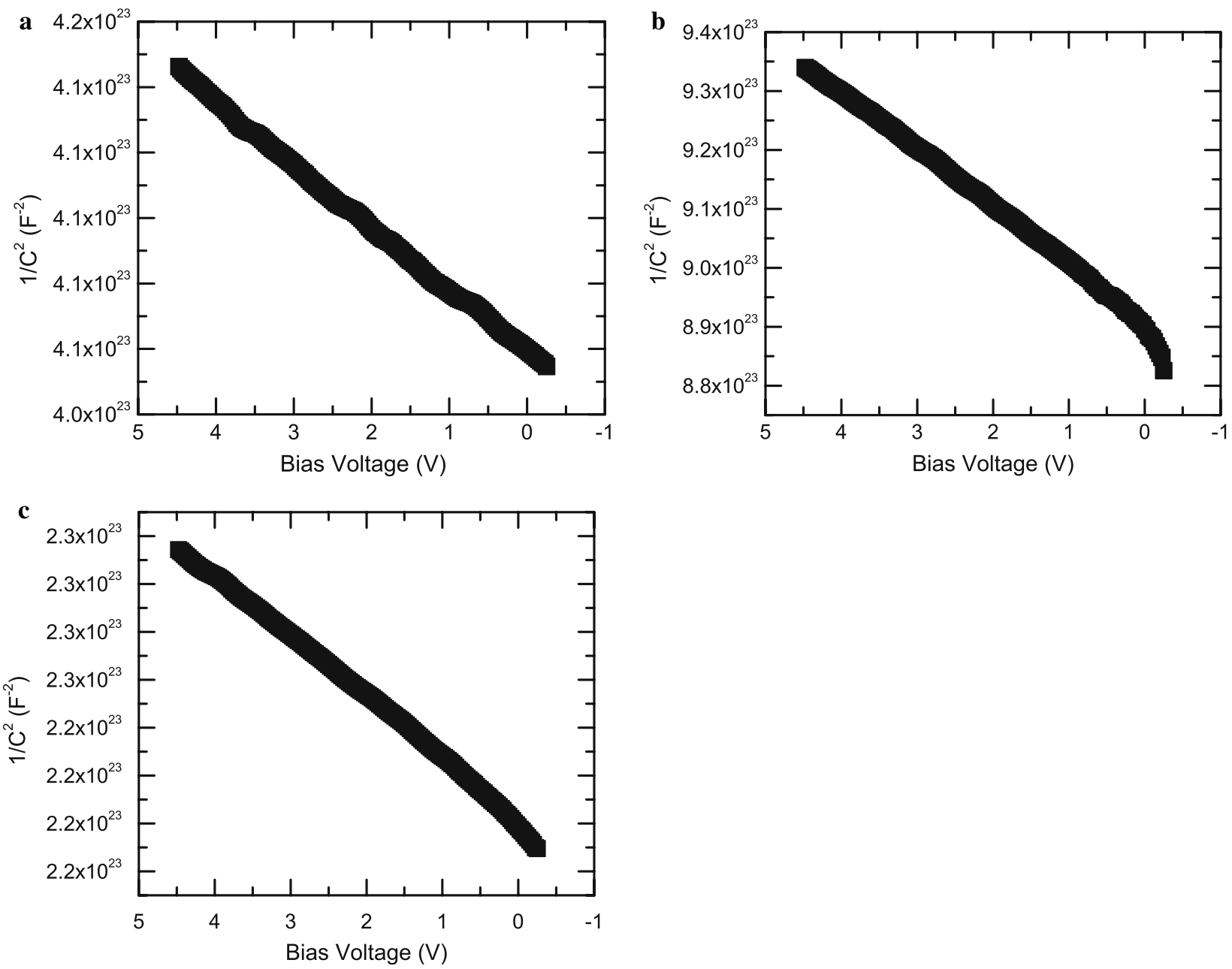

Fig. 2. The $1 / C^{2}$ as a function of bias for a Schottky structure (a) on untreated surface, (b) on surface etched by PEC oxidation at $6 \mathrm{~V}$ for $120 \mathrm{~min}$, and (c) on surface etched as in (b) and annealed as described in the text.

roughness of the as-grown sample was $0.3 \mathrm{~nm}$. As measured from a sample that was oxidized for $2 \mathrm{~h}$ with $6 \mathrm{~V}$ bias, the RMS roughness of the etched surface was $15.6 \mathrm{~nm}$ and the RMS roughness of the etched surface after annealing was $10.3 \mathrm{~nm}$. The increased roughness of the etched surface indicates that the oxidation process is not diffusion limited despite the presence of an oxide cover. This indicates that our process of oxidation likely has some deficiencies manifesting itself in the nonsaturating etch depths and the increase in roughness. It is also possible that the increased roughness is related to the defects in the material being etched. ${ }^{18}$

The relative percentages of atoms on the surface given by AES are shown in Table III. It is seen that oxidation results in a surface without any nitrogen content. After the removal of the oxide, the atomic percentage of oxygen on the surface is very close (within $2 \%$ ) to the levels in the original sample. It can also be seen that annealing of the etched sample did not create a significant change in atomic percentages.

Table III. Atomic Concentration Percentages as Measured by Auger Electron Spectroscopy on Samples at Various Stages of the Etching Process.

\begin{tabular}{|c|c|c|c|c|c|c|}
\hline & $\mathbf{G a}$ & $\mathbf{N}$ & $\mathbf{O}$ & $\mathbf{C}$ & $\mathbf{S}$ & $\overline{\mathrm{Cl}}$ \\
\hline As grown & 40.1 & 40.3 & 10 & 8.1 & 1.5 & - \\
\hline Oxidized & 34.4 & - & 58.8 & 6.7 & - & - \\
\hline Oxide etched & 37.9 & 37.9 & 11.9 & 11.9 & - & 0.4 \\
\hline Oxide etched and annealed & 34.4 & 32.3 & 12.8 & 19.7 & 0.5 & 0.3 \\
\hline
\end{tabular}




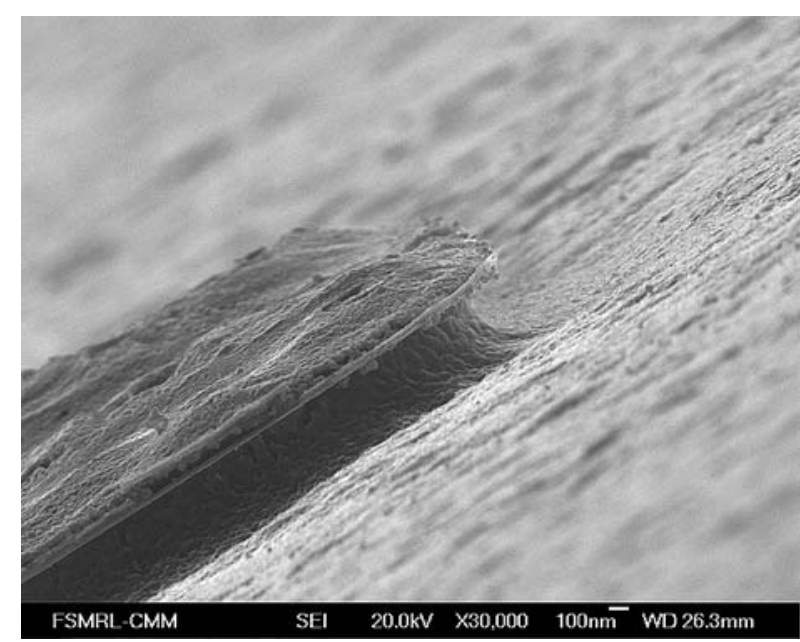

Fig. 3. An SEM image of the Ti/Au/Ni/Au metallization undercut by $\mathrm{BPECO} / \mathrm{DI}$ etching. For this specific sample, the oxidation was performed for $60 \mathrm{~min}$ under $10 \mathrm{~V}$ bias.

The reduction of the roughness after annealing may be a factor in the improvement of the Schottky characteristics seen with annealing. However, the $\mathrm{C}-\mathrm{V}$ and AES results indicate that the improvement is more likely to be related to the removal of point defects. The reduction of the RMS roughness observed with the annealing may be attributed to decomposition of the oxide that remains on the surface after oxide etching. However, no change in oxygen ratio is observed with AES. So, the mechanism by which the annealing results in smoothing of the etched surface remains unclear.

The undercutting of annealed $\mathrm{Ti} / \mathrm{Al} / \mathrm{Ni} / \mathrm{Au}$ ohmic pads was investigated by scanning electron microscopy. A representative image is shown in Fig. 3, where the undercutting of the metallization is clearly observed. The roughness observed on top of the metallization is typical of $\mathrm{Ti} / \mathrm{Al} / \mathrm{Ni} / \mathrm{Au}$ and other Ti/Al-based ohmic contacts to GaN. The roughness of the etched surface can also be seen in Fig. 3.

\section{CONCLUSIONS}

We have demonstrated good quality Schottky contacts on surfaces etched $120 \mathrm{~nm}$ using the BPECO/DI process. The results presented here are the first experiments reporting good quality Schottky devices on GaN surfaces etched more than a few tens of nanometers by any method. The good quality of the Schottky contacts indicates that lowdamage etching of GaN surfaces can be achieved by the BPECO/DI process. Annealing of the etched surfaces at $700^{\circ} \mathrm{C}$ for $30 \mathrm{~s}$ in nitrogen resulted in successful removal of the etch damage. After this anneal, the Schottky breakdown voltages approach values expected from the reduced thickness of the uGaN layer. Possible explanations for the cause of the changes observed in the leakage current, C-V characteristics, and the ideality factor were also discussed. The roughening that results from the $\mathrm{BPECO} / \mathrm{DI}$ oxidation and etching is not clearly understood and is likely to be related to deficiencies in our specific equipment. This will be investigated further.

\section{ACKNOWLEDGEMENTS}

This work was supported by TUBITAK Research Grant No. 104E012. The authors also gratefully acknowledge the support from an MRL research project by which the AES and AFM study was carried out in the Center for Microanalysis of Materials, University of Illinois, which is partially supported by the U.S. Department of Energy, under Grant No. DEFG02-91-ER45439. The authors thank Professors Yalçın Elerman, Sefik Süzer, and Dr. Atilla Aydınlı for helpful discussions.

\section{REFERENCES}

1. S.J. Pearton, J.C. Zolper, R.J. Shul, and F. Ren, J. Appl. Phys. 86, 1 (1999).

2. C.H. Chen, S. Keller, E.D. Haberer, L. Zhang, P. DenBaars, E.L. Hu, U.K. Mishra, and Y. Wu, J. Vac. Sci. Technol. B 17, 2755 (1999).

3. X.A. Cao, J. Pearton, G.T. Dang, A.P. Zhang, F. Ren, and J.M. Hove, IEEE Trans. Electron. Dev. 47, 1320 (2000).

4. F.A. Khan, L. Zhou, V. Kumar, and I. Adesida, J. Vac. Sci. Technol. B 19, 2926 (2001).

5. D. Zhuang and J.H. Edgar, Mar. Sci. Eng. R 48, 1 (2005).

6. C. I. L.T. G. Youtsey Adesida Romana Bulman, Appl. Phys. Lett. 72, 560 (1998).

7. T Rotter, et al., Electron. Lett. 37, 715 (2001).

8. J.E. Borton, C. Cai, M.I. Nathan, P. Chow, J.M. Van Hove, A. Wowchak, and H. Morkoc, Appl. Phys. Lett. 77, 1227 (2000).

9. B. Yang and P. Fay, J. Vac. Sci. Technol. B 22, 1750 (2004).

10. A.T. Ping, D. Selvanathan, C. Youtsey, E. Piner, J. Redwing, and I. Adesida, Electron. Lett. 35, 2140 (1999).

11. J.W. Seo, C.S. Oh, H.S. Jeong, J.W. Yang, K.Y. Lim, C.J. Yoon, and H.J. Lee, Appl. Phys. Lett. 81, 1029 (2002).

12. E.J. Miller, E.T. Yu, P. Waltereit, and J.S. Speck, Appl. Phys. Lett. 84, 535 (2004).

13. J.W.P. Hsu, M.J. Manfra, R.J. Molnar, B. Heying, and J.S. Speck, Appl. Phys. Lett. 81, 79 (2002).

14. P. Pipinys and V. Lapeika, J. Appl. Phys. 99, 093709 (2006).

15. S. Limpijumnong and C.G. Van de Walle, Phys. Rev. B 69, 035207 (2004).

16. C.G. Van de Walle and J. Neugebauer, J. Appl. Phys. 95, 3851 (2004).

17. J.M. Hwang, J.T. Hsieh, H.L. Hwang, and W.H. Hung, MRS Int. J. Nitride Semicond. Res. 5, W11.73(2000).

18. C. Youtsey, L.T. Romano, and I. Adesida, Appl. Phys. Lett. 73, 797 (1998). 